\title{
Hemodynamic response to orthostatic stress immediately after dialysis session in chronic hemodialysis patients
}

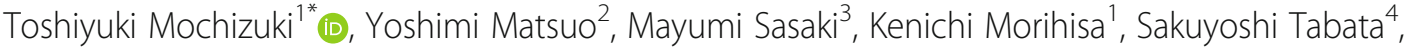 \\ Toyofumi Fukuda ${ }^{5}$ and Ibuki Yajima ${ }^{5}$
}

\begin{abstract}
Orthostatic hypotension is a risk factor for falls among chronic hemodialysis (HD) patients. This study aimed to investigate the hemodynamic response to orthostatic stress immediately after an HD session. Twenty-one HD patients (mean age, $70.2 \pm 8.1$ years; $\mathrm{HD}$ duration, $7.5 \pm 6.4$ years) participated in this study. Each 5-min hemodynamic monitoring was performed in the supine, semi-recumbent, and sitting positions immediately after an HD session. Hemodynamic variables were measured using a noninvasive beat-to-beat monitoring device during the test. Patients were divided into an intradialytic hypotension (IDH) group and a non-IDH group according to the presence or absence of IDH on the day of the measurements, and intra- and intergroup comparisons were performed. In the IDH group, the nadir values of systolic blood pressure in the semi-recumbent and sitting positions were significantly lower than those in the supine position and the last systolic blood pressure in the semi-recumbent position. The nadir of stroke volume in the sitting position was significantly lower than that in the supine position. There were no statistically significant intergroup differences in the changes for any positions. These results suggest that patients with IDH require special attention when getting out of bed to prevent post-dialytic falls.
\end{abstract}

Keywords: Hemodialysis, Hypotension, Noninvasive continuous hemodynamic monitoring, Postural change

\section{Background}

Orthostatic hypotension $(\mathrm{OH})$ is caused by an excessive decrease in cardiac output (CO) and/or compensatory vasoconstrictor mechanisms such as autonomic failure and age-related physiologic changes [1]. $\mathrm{OH}$ is a risk factor for falls among chronic hemodialysis (HD) patients. The cross-sectional prevalence of $\mathrm{OH}$ in $\mathrm{HD}$ patients is reportedly higher than that in the general population. Previous studies reported a $42 \%$ prevalence of $\mathrm{OH}$ in the introductory phase of HD [2] versus $5-30 \%$ in unselected individuals aged $\geq 65$ years [3].

In the post-dialysis period, there are several HD-related risk factors for $\mathrm{OH}$ such as the rapid removal of intravascular volume and several hours of bed rest during HD therapy, in addition to other common risk factors such as advanced age, autonomic failure, and frailty. Previous

\footnotetext{
* Correspondence: ekisaimochizuki@yahoo.co.jp

${ }^{1}$ Physical Therapy Service, Ibuki Clinic, 1-37 Kofuku-cho, Kadoma, Osaka 571-0066, Japan

Full list of author information is available at the end of the article
}

studies demonstrated that the incidences of $\mathrm{OH}$ and falls were higher in the post- than pre-dialysis. Roberts et al. reported that $69.6 \%$ of patients had post-dialysis $\mathrm{OH}$ versus $34.8 \%$ with pre-dialysis $\mathrm{OH}$ [4]. The incidences of falls pre- and post-dialysis session are reportedly 9-41\% [5] and $27-73 \%$ [6], respectively. These findings suggest the risk for post-dialytic falls. Furthermore, post- $\mathrm{HD} \mathrm{OH}$ and intradialytic hypotension (IDH) are significant and independent factors that affect mortality in HD patients [7]. Therefore, studying hemodynamic responses to orthostatic stress after dialysis is important to manage the fall risk in the care for HD patients. However, to our knowledge, little is known about hemodynamic response during postural change immediately after an HD session.

A manual sphygmomanometer is most frequently used to measure $\mathrm{OH}$; however, this device provides only a single blood pressure value unless repeated measurements are taken to identify clinically relevant $\mathrm{OH}$ [8]. Continuous beat-to-beat blood pressure measurements are increasingly available in hospital settings [9]. Beat-to-beat 
methods have been validated against intra-arterial recordings, meet acceptable criteria for bias and precision [10], and identified a higher prevalence of $\mathrm{OH}$ than sphygmomanometry [11]. Furthermore, beat-to-beat technology is reportedly more effective for detecting $\mathrm{OH}$ and discriminating blood pressure responses in fallers and non-fallers compared to intermittent manual sphygmomanometer assessments [12].

This study aimed to investigate hemodynamic responses to orthostatic stress immediately after an HD session using continuous beat-to-beat blood pressure measurements. We hypothesized that the decreases in blood pressure upon orthostatic stress immediately after an HD session would be larger in patients with IDH than in those without IDH.

\section{Methods}

\section{Patients}

This observational study was conducted in a single dialysis center (Ibuki Clinic, Osaka, Japan). Twenty-five HD patients (mean age, $70.8 \pm 8.6$ years; male/female ratio, 10/15) who underwent maintenance HD three times a week at Ibuki Clinic, Osaka, Japan, participated in this study. None of the patients had common risk factors for falls such as gait disorder, visual impairment, or neurologic disease [13], and all were able to walk into the clinic unassisted and had a history of hypotensive episodes immediately after HD session within the previous year. We excluded patients with severe heart disease (New York Heart Association III or IV) or peripheral artery disease (Fontaine III or IV) as well as those with neurological diseases that induce $\mathrm{OH}$.

This study was approved by the Ethics Committee of Mukogawa Women's University, and written informed consent was obtained from each participant after a detailed explanation of the study protocol was provided.

\section{Demographic and clinical characteristics}

The demographic and clinical characteristics obtained from patients' medical records included age, sex, body mass index, presence of underlying diabetes mellitus (DM), HD vintage, dry weight, cardiothoracic ratio, left ventricular ejection fraction (LVEF) by echocardiography, and hemoglobin $(\mathrm{Hb})$, hematocrit $(\mathrm{Ht})$, blood urea nitrogen, creatinine, albumin (Alb), total cholesterol, triglyceride, high-density lipoprotein cholesterol, and C-reactive protein levels in a blood sample obtained before HD. Serum B-type natriuretic peptide (BNP) level was measured before and after the HD session. Parameters concerning arterial stiffness included ankle-brachial index (ABI), toe-brachial index, brachial-ankle pulse wave velocity, and skin perfusion pressure. The body fluid removal rate (\%) during the HD session was assessed according to the following equation: body fluid removal rate $(\%)=($ pre-dialysis body weight - post-dialysis body weight)/post-dialysis body weight $\times 100$ [14] .

\section{Study protocol}

Hemodynamic monitoring was performed on the day of the HD session after a 1-day interval at the timing of getting out of the dialysis bed for returning home. Arterial blood pressure was recorded every 30 min during the HD session by the cuff method with an automatic blood pressure monitor. IDH was defined as a decrease of $\geq$ $20 \mathrm{mmHg}$ in systolic blood pressure (SBP) during the HD session [15]. Patients were divided into an IDH group and a non-IDH group according to the aforementioned definition of IDH on the day of the measurement.

Immediately after the HD session and detachment of the dialysis circuit, subjects were maintained in the supine position in the dialysis bed for $5 \mathrm{~min}$ and then passively moved into a semi-recumbent position by repositioning the head of the bed to $60^{\circ}$ and maintained the position for $5 \mathrm{~min}$ (semi-recumbent position phase). Next, the subjects were asked to move unassisted into a chair placed beside the dialysis bed and remain there for 5 min (chair sitting position phase). Each postural change occurred within $10 \mathrm{~s}$.

\section{Hemodynamics measurement}

During the aforementioned postural changes, we continuously recorded noninvasive beat-to-beat arterial blood pressure (Portapres, Finapres Medical Systems, Amsterdam, The Netherlands) from the pointer or middle finger of the hand without vascular access. The finger cuff of the Portapres was sized to each participant per the manufacturer's specifications after a calibration procedure. Participant height, weight, and age were entered before starting the device to ensure accurate determination of stroke volume (SV) and CO using the Modelflow algorithm (Modelflow, TNO Biomedical Instrumentation, Amsterdam, The Netherlands) [16]. A hydrostatic height correction sensor was placed at the level of the heart to compensate for any hand movements relative to the heart that could impact the blood pressure recordings.

\section{Assessment of hemodynamics}

SBP and diastolic blood pressure (DBP) were derived from the arterial pressure waveform. Heart rate (HR) was computed as the inverse of the inter-beat interval and expressed as beats per minute. Beat-to-beat left ventricular SV was estimated by modeling flow from the arterial pressure waveform (16). CO was the product of estimated SV and HR. Total peripheral resistance (TPR) was computed by mean blood pressure at the heart level divided by the computed $\mathrm{CO}$. 


\section{Data processing}

The data were exported to Microsoft Excel spreadsheets with BeatScope software using the 5-s average method [9], which was reported to show the best association with fall history using various mean times.

\section{Outcome measures}

SBP measurements at the following time points were analyzed:

1. Baseline: SBP of the final 5-s average of the supine position phase before the semi-recumbent position phase

2. Semi-recumbent nadir: the lowest 5-s average SBP obtained within the first $3 \mathrm{~min}$ of the semi-recumbent position phase

3. Semi-recumbent recovery: SBP of the final 5-s average of the semi-recumbent position phase before the chair sitting position phase

4. Sitting nadir: lowest 5-s average SBP obtained within the first 3 min of the chair sitting position phase

Values for DBP, HR, SV, CO, and TPR at the same time point of SBP obtained according to the above definition were used in the analysis. Data acquired during postural changes were excluded to avoid any effect from body motion.

\section{Statistical analysis}

Values for SBP, DBP, HR, SV, CO, and TPR between each time point were analyzed by two-way analysis of variance with the repeated measures or Kruskal-Wallis and Bonferroni test as the post hoc test in each group. The two groups in each time point were compared using an unpaired $t$ test or the Mann-Whitney test after converting to the difference from the baseline values (i.e., $\triangle \mathrm{SBP}, \triangle \mathrm{DBP})$ and percentage changes relative to the baseline values (i.e., \%HR, \%SV, \%CO, \%TPR). Clinical characteristics in each group were compared using an unpaired $t$ test or the Mann-Whitney test or chi-square test. Values are shown as mean $\pm \mathrm{SD}$. $P$ values $<0.05$ were considered statistically significant. All statistical analyses were performed using IBM SPSS version 19 (IBM Japan Inc., Tokyo, Japan).

\section{Results}

\section{Clinical and demographic characteristics}

We evaluated 21 patients (mean age, $70.3 \pm 8.0$; male/female ratio, 9/12); three patients were excluded before the hemodynamic monitoring because of discomfort including nausea just after the HD session. In the three patients, SBP decreased by $\geq 20 \mathrm{mmHg}$ in two patients and by $<20 \mathrm{mmHg}$ in one patient during the HD session. Another patient was excluded because of syncope with SBP decrease of $\geq 20 \mathrm{mmHg}$ during the HD session. The underlying kidney diseases were diabetic nephropathy $(n=15)$, chronic glomerulonephritis $(n=4)$, polycystic kidney disease $(n=1)$, and chronic nephritis $(n=1)$. The body fluid removal rate during the HD in all participants was $<5 \%$. No patient was administered midodrine to prevent IDH before and during the HD session or complained of hypotensive symptoms including dizziness and faintness during the hemodynamic monitoring. Eleven patients were categorized into the IDH group, and the other 10 were categorized into the non-IDH group.

Clinical and demographic characteristics for each group are shown in Table $1 . \mathrm{Hb}$ and $\mathrm{Ht}$ levels in the IDH group were significantly higher than those in the non-IDH group ( $P=0.009$ and $P=0.033$, respectively). The mean Alb level in the IDH group was significantly lower than that in the non-IDH group $(P=0.040)$. There were no significant intergroup differences in cardiac function markers or body fluid removal rates (Table 2).

\section{Hemodynamics during postural changes}

The changes of hemodynamic parameters between each time point are shown in Fig. 1. SBP for semi-recumbent nadir in the IDH group were significantly lower than those for baseline and semi-recumbent recovery. The SBP for sitting nadir in the IDH group was significantly lower than that for semi-recumbent recovery. The SV for sitting nadir in the IDH group was significantly lower than that for baseline.

There were no statistically significant intergroup differences between the two groups in changes at any time point.

\section{Discussion}

In the present study, hemodynamic monitoring during postural changes was performed immediately after an HD session in both the semi-recumbent and sitting positions using noninvasive beat-to-beat blood pressure monitoring. $\mathrm{OH}$ has been traditionally measured on active standing or on a head-up tilt table at an angle of $60^{\circ}$. In patients who are unable to stand or who are at risk for symptomatic standing postural hypotension, evaluating postural hypotension in a sitting position is recommended. Advanced age, significant bed rest, and hypertension are reportedly the most important predisposing factors for seated postural hypotension [1]. The effectiveness of the orthostatic stress test in the sitting position was demonstrated in a study by Shaw et al., in which passive seated orthostatic stress and head-up tilt testing produced blood pressure responses of similar magnitudes and no difference was seen in cerebral blood 
Table 1 Characteristics of subjects

\begin{tabular}{|c|c|c|c|}
\hline & $\begin{array}{l}\text { IDH group } \\
(n=11)\end{array}$ & $\begin{array}{l}\text { Non-IDH group } \\
(n=10)\end{array}$ & $P$ value \\
\hline \multicolumn{4}{|l|}{ Characteristics } \\
\hline Age (years) & $71.1 \pm 8.7$ & $70.4 \pm 7.1$ & 0.852 \\
\hline HD vintage (years) & $7.5 \pm 5.6$ & $7.4 \pm 7.2$ & 0.888 \\
\hline Male/female & $5 / 6$ & $4 / 6$ & 0.617 \\
\hline $\mathrm{DM}(+/-)$ & $8 / 3$ & $7 / 3$ & 0.705 \\
\hline $\mathrm{BMI}$ & $23.4 \pm 2.4$ & $22.2 \pm 3.2$ & 0.354 \\
\hline $\mathrm{CTR}$ & $51.1 \pm 3.4$ & $49.2 \pm 3.4$ & 0.246 \\
\hline \multicolumn{4}{|l|}{ Medications } \\
\hline ACEI/ARB (\%) & 9.1 & 10.0 & 0.739 \\
\hline$\beta$-blocker (\%) & 9.1 & 10.0 & 0.739 \\
\hline Ca antagonist (\%) & 36.4 & 20.0 & 0.145 \\
\hline \multicolumn{4}{|l|}{ Laboratory parameters } \\
\hline $\mathrm{Hb}$ & $11.8 \pm 0.6$ & $10.6 \pm 1.2$ & 0.009 \\
\hline $\mathrm{Ht}$ & $36.3 \pm 2.3$ & $32.7 \pm 4.4$ & 0.033 \\
\hline BUN & $56.3 \pm 17.7$ & $59.2 \pm 13.1$ & 0.689 \\
\hline $\mathrm{Cr}$ & $9.79 \pm 4.41$ & $9.23 \pm 1.23$ & 0.369 \\
\hline Alb & $3.4 \pm 0.3$ & $3.6 \pm 0.2$ & 0.040 \\
\hline $\mathrm{TC}$ & $163.1 \pm 43.9$ & $171.3 \pm 37.9$ & 0.669 \\
\hline TG & $109.3 \pm 48.8$ & $104.3 \pm 35.6$ & 0.815 \\
\hline $\mathrm{HDL}-\mathrm{C}$ & $42.9 \pm 11.4$ & $42.8 \pm 10.6$ & 0.983 \\
\hline CRP & $0.24 \pm 0.23$ & $0.09 \pm 0.08$ & 0.128 \\
\hline \multicolumn{4}{|c|}{ Atherosclerotic parameters } \\
\hline$A B \mid$ & $0.95 \pm 0.21$ & $1.10 \pm 0.13$ & 0.087 \\
\hline $\mathrm{TB} \mid$ & $0.59 \pm 0.15$ & $0.63 \pm 0.13$ & 0.596 \\
\hline ba-PWV & $2233.3 \pm 460.0$ & $2087.1 \pm 584.4$ & 0.530 \\
\hline SPP & $63.3 \pm 16.1$ & $63.1 \pm 11.9$ & 0.979 \\
\hline
\end{tabular}

Data are expressed as means $\pm S D$

$B M I$ body mass index, DM diabetes mellitus, CTR cardiothoracic ratio, $A C E I$ angiotensin-converting-enzyme inhibitor, $A R B$ angiotensin receptor blocker, $\mathrm{Ca}$ calcium, $\mathrm{Hb}$ hemoglobin, $\mathrm{Ht}$ hematocrit, $\mathrm{BUN}$ blood urea nitrogen, $\mathrm{Cr}$ creatinine, Alb albumin, TC total cholesterol, TG triglycerides, HDL-c highdensity lipoprotein cholesterol, CRP C-reactive protein, $A B I$ ankle-brachial index, TBI toe-brachial index, ba-PWV brachial-ankle pulse wave velocity, SPP skin perfusion pressure

Table 2 Markers of cardiac function and removed body fluid rate

\begin{tabular}{llll}
\hline & $\begin{array}{l}\text { IDH group } \\
(n=11)\end{array}$ & $\begin{array}{l}\text { Non-IDH group } \\
(n=10)\end{array}$ & $P$ value \\
\hline LVEF (\%) & $67.8 \pm 7.8$ & $71.1 \pm 12.5$ & 0.495 \\
BNP (pre-HD session) & $177.7 \pm 120.4$ & $109.4 \pm 67.9$ & 0.160 \\
BNP (post-HD session) & $83.7 \pm 53.6$ & $61.4 \pm 30.8$ & 0.297 \\
Removed body fluid rate (\%) & $3.48 \pm 1.07$ & $2.96 \pm 0.45$ & 0.187 \\
\hline
\end{tabular}

flow velocity in the middle cerebral artery between the two tests in healthy volunteers [8].

In this study, patients with IDH had orthostatic intolerance upon postural change from a supine to a semi-recumbent position immediately after an HD session. This finding supports our assumption that HD therapy negatively affected orthostatic tolerance in HD patients with IDH. Furthermore, the nadir SBP in the sitting position was significantly lower than that in the semi-recumbent position in the patients with IDH, that is, the change from the semi-recumbent to the sitting position decreased the SBP.

In the IDH group, compensatory responses to orthostatic stress such as increases in HR and TPR for stabilizing arterial pressure did not reach statistical significance in spite of significant SBP and SV decrease. Since the orthostatic HR response may be a useful marker of cardiac baroreflex function [10], this result suggests that autonomic dysfunction including diabetic and/or uremic neuropathy was a possible factor for those poor responses considering the lack of a significant intergroup difference in major risk factors for orthostatic intolerance such as age, cardiac function measured by LVEF and BNP level, and body fluid removal rate. Further studies are needed to investigate the effect of those factors on orthostatic intolerance immediately after an HD session.

The post-dialysis period includes not only a higher risk for postural hypotension but also other factors that may increase the risk for accidental falls. Cook et al. revealed that HD treatment altered motor function in older HD patients, weakening their strength and decreasing their mobility after the HD session [7]. Soangra et al. reported decreased sit-to-walk test performance pre- versus post-HD session [17]. Furthermore, a greater postural sway in the post- versus pre-HD session was observed [18] in addition to poorer postural control in HD patients than in age-matched controls, which was further exacerbated by the simultaneous performance of a cognitive task [19]. These results suggest that the immediate post-HD period is particularly unsafe, so medical staff in dialysis units should be sure to provide the necessary support [18]. Accordingly, dialysis staff should consider such alterations concerning physical function post-dialysis as well as the probability of the aforementioned postural hypotension immediately after the HD session. It is likely that dialysis staff already knows about the increased risk for post-dialysis falls; however, only a few studies have reported hemodynamic data from that period. This study is the first to use beat-to-beat technology immediately after an HD session to capture rapid and transient hemodynamic fluctuations.

There were significant differences in $\mathrm{Hb}, \mathrm{Ht}$, and $\mathrm{Alb}$ levels between the IDH and non-IDH groups. It is 

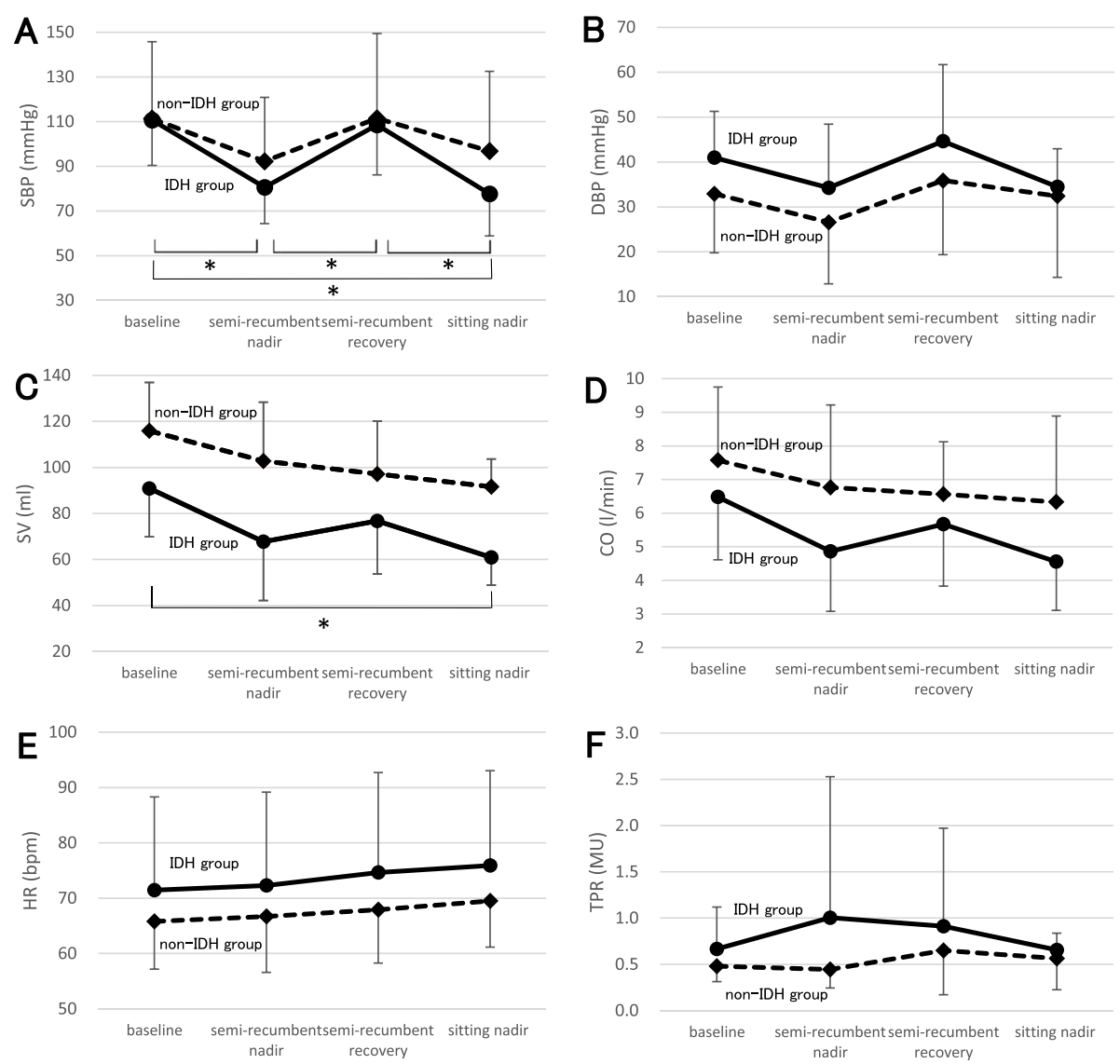

Fig. 1 Hemodynamic responses to postural changes immediately after dialysis session. a SBP. b DBP. c SV. d CO. e HR. f TPR. Data are expressed as means \pm SD. ${ }^{*} P<0.05$

known that anemia is one of the factors that aggravate $\mathrm{OH}$ in the general population [20]. However, $\mathrm{Hb}$ and $\mathrm{Ht}$ level measurements in both groups in this study were within the target range recommended by the Japanese Society of Dialysis Therapy guideline [21], which suggests that even patients with target $\mathrm{Hb}$ and $\mathrm{Ht}$ levels could develop $\mathrm{OH}$ immediately after HD. Meanwhile, the K/DOQI guidelines [15] include hypoalbuminemia as a factor related to IDH; however, whether the intergroup difference in Alb has clinically relevant significance remains unclear because of a lack of evidence.

There are several limitations to this study. First, the small sample size may have affected its ability to show a statistically significant difference in some measures. In addition, we could not evaluate the impact of DM which is the most common disease associated with peripheral damage to the autonomic nerves [22] and could not evaluate patients with hypotensive symptoms before hemodynamic monitoring, who might have had orthostatic intolerance just after HD session. Second, the correlation between IDH and orthostatic intolerance immediately after dialysis was not fully clarified. It is well known that intradialytic and orthostatic hypotension have multifactorial causes. Future studies to identify factors related to post-dialysis $\mathrm{OH}$ are needed to enable the earlier detection of fall risk. Third, there is a diverse pattern of orthostatic BP decay [11] such as initial and delayed $\mathrm{OH}$ in which a blood pressure decrease occurs within $15 \mathrm{~s}$ and after $3 \mathrm{~min}$, respectively [23]; however, we could not investigate the blood pressure patterns and recovery times. Further studies on blood pressure decrease timing are needed to develop more effective strategies to reduce the probability of post-dialytic falls in HD patients upon returning home.

\section{Conclusion}

In patients with IDH, postural changes from the supine to the semi-recumbent position immediately after an HD session may significantly decrease SBP. Postural changes from a semi-recumbent to a sitting position may also significantly decrease SBP. These results suggest that beat-to-beat hemodynamic monitoring during HD session provides useful information to identify the patients with a potential risk for post-dialytic falls and that dialysis care team members should provide support to patients with IDH who are transferring out of bed to prevent post-dialytic falls. 


\section{Abbreviations}

ABI: Ankle-brachial index; Alb: Albumin; BNP: B-type natriuretic peptide; CO: Cardiac output; DBP: Diastolic blood pressure; DM: Diabetes mellitus; Hb: Hemoglobin; HD: Hemodialysis; HR: Heart rate; Ht: Hematocrit; IDH: Intradialytic hypotension; LVEF: Left ventricular ejection fraction; OH: Orthostatic hypotension; SBP: Systolic blood pressure; SV: Stroke volume; TPR: Total peripheral resistance

\section{Acknowledgements}

The authors thank the hospital staff and patients who participated in this study.

\section{Funding}

The authors declare that there is no funding related to this manuscript.

\section{Availability of data and materials}

The datasets analyzed during the present study are available from the corresponding author on reasonable request.

\section{Authors' contributions}

TM and YM were involved in the study design and article writing of the manuscript. TM, MS and KM participated in the study procedure implementation and data collection. ST, TF, and IY kindly reviewed and revised the manuscript. All authors read and approved the final manuscript

\section{Ethics approval and consent to participate}

This study was approved by the Ethical Committee of Mukogawa Women's University, and written informed consent was obtained from each participant after a detailed explanation of the study protocol was provided.

\section{Consent for publication}

Not applicable.

\section{Competing interests}

The authors declare that they have no competing interests.

\section{Publisher's Note}

Springer Nature remains neutral with regard to jurisdictional claims in published maps and institutional affiliations.

\section{Author details}

'Physical Therapy Service, Ibuki Clinic, 1-37 Kofuku-cho, Kadoma, Osaka 571-0066, Japan. ${ }^{2}$ Graduate School of Health and Sports Sciences, Mukogawa Women's University, 6-46 Ikebiraki, Nishinomiya, Hyogo 663-8558, Japan. ${ }^{3}$ Nursing Department, Ibuki Clinic, 1-37 Kofuku-cho, Kadoma, Osaka 571-0066, Japan. ${ }^{4}$ Department of Internal Medicine, Ibuki Clinic, 1-37 Kofuku-cho, Kadoma, Osaka 571-0066, Japan. ${ }^{5}$ Department of Urology, Ibuki Clinic, 1-37 Kofuku-cho, Kadoma, Osaka 571-0066, Japan.

\section{Received: 31 August 2018 Accepted: 29 October 2018}

Published online: 21 November 2018

\section{References}

1. Gorelik O, Cohen N. Seated postural hypotension. J Am Soc Hypertens. 2015;12:985-92.

2. Sasaki O, Nakahama H, Nakamura S, et al. Orthostatic hypotension at the introductory phase of haemodialysis predicts all-cause mortality. Nephrol Dial Transplant. 2005:20:377-81.

3. Low PA. Prevalence of orthostatic hypotension. Clin Auton Res. 2008; 18(Suppl 1):8-13.

4. Roberts RG, Kenny RA, Brierley EJ. Are elderly haemodialysis patients at risk of falls and postural hypotension? Int Urol Nephrol. 2003;35:415-21.

5. Polinder-Bos HA, Emmelot-Vonk MH, Gansevoort RT, Diepenbroek A, Gaillard CA. High fall incidence and fracture rate in elderly dialysis patients. Neth J Med. 2014;72:509-15.

6. Cook WL, Tomlinson G, Donaldson M, et al. Falls and fall-related injuries in older dialysis patients. Clin J Am Soc Nephrol. 2006:1:1197-204.

7. Shoji T, Tsubakihara Y, Fujii M, Imai E, et al. Hemodialysis-associated hypotension as an independent risk factor for two-year mortality in hemodialysis patients. Kidney Int. 2004;66:1212-20.
8. Shaw BH, Loughin TM, Mackey DC, Robinovitch SN, Claydon VE. The effect of orthostatic stress type on cardiovascular control. Blood Press Monit. 2014 19:327-38.

9. van der Velde $\mathrm{N}$, van den Meiracker $\mathrm{AH}$, Stricker $\mathrm{BH}$, van der Cammen TJ. Measuring orthostatic hypotension with the Finometer device: is a blood pressure drop of one heartbeat clinically relevant? Blood Press Monit. 2007;12:167-71.

10. Shaw BH, Claydon VE. The relationship between orthostatic hypotension and falling in older adults. Clin Auton Res. 2014;24:3-13.

11. Cooke J, Carew S, Quinn C, et al. The prevalence and pathological correlates of orthostatic hypotension and its subtypes when measured using beat-tobeat technology in a sample of older adults living in the community. Age Ageing. 2013;42:709-14

12. Shaw BH, Loughin TM, Robinovitch $\mathrm{SN}$, et al. Cardiovascular responses to orthostasis and their association with falls in older adults. BMC Geriatr. 2015;15:174.

13. Abdel-Rahman EM, Turgut F, Turkmen K, Balogun RA. Falls in elderly hemodialysis patients. QJM. 2011;104:829-38.

14. Yamamoto K, Kobayashi N, Kutsuna T, et al. Excessive fall of blood pressure during maintenance hemodialysis in patients with chronic renal failure is induced by vascular malfunction and imbalance of autonomic nervous activity. Ther Apher Dial. 2012;16:219-25.

15. KJOQI workgroup. K/DOQI clinical practice guidelines for cardiovascular disease in dialysis patients. Am J Kidney Dis. 2005:45(Suppl 3):S1-153.

16. Wesseling KH, Jansen JR, Settels JJ, Schreuder JJ. Computation of aortic flow from pressure in humans using a nonlinear, three-element model. J Appl Physiol. 1993;74:2566-73.

17. Soangra R, Lockhart TE, Lach J, Abdel-Rahman EM. Effects of hemodialysis therapy on sit-to-walk characteristics in end stage renal disease patients. Ann Biomed Eng. 2013;41:795-805.

18. Magnard J, Lardy J, Testa A, Hristea D, Deschamps T. The effect of hemodialysis session on postural strategies in older end-stage renal disease patients. Hemodial Int. 2015;19:553-61.

19. Shin S, Chung HR, Fitschen PJ, et al. Postural control in hemodialysis patients. Gait Posture. 2014:39:723-7.

20. Figueroa JJ, Basford JR, Low PA. Preventing and treating orthostatic hypotension: as easy as A, B, C. Cleve Clin J Med. 2010;77:298-306.

21. Yamamoto H, Nishi S, Tomo T, et al. 2015 Japanese Society for Dialysis Therapy: guidelines for renal anemia in chronic kidney disease (in Japanese). Touseki Kaishi. 2016;49:89-158

22. Shibao C, Lipsitz LA, Biaggioni I. American Society of Hypertension Writing Group. Evaluation and treatment of orthostatic hypotension. J Am Soc Hypertens. 2013;7:317-24.

23. Freeman $\mathrm{R}$, Wieling $\mathrm{W}$, Axelrod FB, et al. Consensus statement on the definition of orthostatic hypotension, neurally mediated syncope and the postural tachycardia syndrome. Clin Auton Res. 2011:21:69-72.
Ready to submit your research? Choose BMC and benefit from:

- fast, convenient online submission

- thorough peer review by experienced researchers in your field

- rapid publication on acceptance

- support for research data, including large and complex data types

- gold Open Access which fosters wider collaboration and increased citations

- maximum visibility for your research: over $100 \mathrm{M}$ website views per year

At $\mathrm{BMC}$, research is always in progress.

Learn more biomedcentral.com/submissions 